\title{
EVALUATION OF A HEADSPACE SOLID-PHASE MICROEXTRACTION WITH DIFFERENT FIBRES FOR VOLATILE COMPOUND DETERMINATION IN SPECIALTY COFFEE BREWS
}

\author{
Ilze Laukalēja, Zanda Krūma \\ Latvia University of Life Sciences and Technologies, Latvia \\ ilze.laukaleja@gmail.com
}

\begin{abstract}
The aroma of coffee is the main quality factor. Compounds with floral, fruity, citrus and sometimes fermented aroma notes are defined as high quality characteristics for the specialty coffee brew. Commercial coffees mostly are at medium roast and the aroma profile is focused on balanced chocolate, caramel, toasted bread and almond bitterness aroma notes. These sensory characteristics set the focus for volatile compound profile analysis by Headspace solidphase microextraction (HS-SPME). The most popular fibres for commercial coffee aroma profile analysis are DVB/ CAR/PDMS, CAR/PDMS, PDMS/DVB and PA. There is limited research done about specialty coffee aroma profile and evaluation of more suitable fibres for solid-phase microextraction. The aim of the research is to evaluate the aroma composition of specialty coffee brews using different SPME fibres. Results demonstrated that CAR/PDMS fibre, compared to other fibres, can extract significantly more volatile compounds with higher peak areas in all chemical compound groups, except phenols. The CAR/PDMS was the only fibre which could detect all 17 important volatile compounds for specialty coffee. In conclusion, from the given research evidence, CAR/PDMS fibre is suggested as the most suitable SPME fibre coating for volatile compound extraction for specialty coffee brew import. The result provides evidence for improved specialty coffee aroma profile analysis by SPME.
\end{abstract}

Key words: specialty coffee, volatile compounds, SPME, fibre coating.

\section{Introduction}

HS-SPME is a simple, rapid but sensitive method for volatile compound extraction from food products and beverages (Chen, Chiang, \& Chung, 2019). There are commercially available 7 SPME coatings: polydimethylsiloxane (PDMS), polyacrylate (PA), carboxen/polydimethylsiloxane (CAR/PDMS), polydimethylsiloxane/divinylbenzene (PDMS/DVB), carbowax/divinylbenzene (CW/DVB), carbowax/ template resin (CW/TR) and divinylbenzene/ carboxen/polydimethylsiloxane (DVB/CAR/PDMS) (Spietelun et al., 2010). It is important to evaluate SPME coatings, extraction and desorption technique for a specific product (Chen, Chiang, \& Chung, 2019). The most popular fibres for commercial coffee aroma profile analysis are DVB/CAR/PDMS, CAR/PDMS, PDMS/DVB and PA. The DVB/CAR/ PDMS fibre was selected for Mestdagh et al. (2014) study about the kinetics of coffee aroma extraction and Lee, Kim, \& Lee (2017) study for coffee brew volatile compound profile determination of reversed coffee grinding and roasting process. The PDMS/ DVB fibre was confirmed in Roberts, Pollien, \& Milo (2000) research as the most suitable SPME fibre coating for wider range coffee volatile compound extraction. Petisca et al. (2012) study suggested CAR/PDMS as a beneficial SPME fibre coating for coffee furan compound detections. The CAR/PDMS fibre also was chosen by Kim et al. (2018) for the prediction of key aroma compounds in differently roasted coffee samples. Commercial coffees aroma profile is focused on balanced chocolate, caramel, toasted bread and almond bitterness aroma notes.
These sensory characteristics set the focus for volatile compound profile by HS-SPME. Specialty coffee focus is on highlighting specific aroma and flavour notes from coffee by high-quality standards. From niche product specialty coffee has become a whole industry. Specialty coffee is defined as high quality coffee (Donnet, Weatherspoon, \& Hoehn, 2008; Lee, Bonn, \& Cho, 2013). Compounds with floral, fruity, citrus and sometimes fermented aroma notes are compared as high quality characteristics for the coffee brew (Fassio et al., 2017; Piccino et al., 2014; Poltronieri \& Rossi, 2016; Silveira et al., 2016).There is limited research done about specialty coffee aroma profile and evaluation of more suitable fibres for solid phase microextraction. The aim of the research is to evaluate the volatile composition of specialty coffee brews using different SPME fibres.

\section{Materials and Methods \\ Coffee samples}

Five samples of coffee (Coffea arabica L.) beans were collected from two different coffee roasteries in Latvia. The main characteristics of the coffee samples are summarized in Table 1. Natural processing method was applied for Honduras_1, Kenya, Columbia, Ethiopia coffee samples, and anaerobic processing method-Honduras_2. All coffees are roasted at lightmedium roast level and rated as specialty coffees: specialty coffee grade applies if the total specialty cup quality score is 80 points or above (Donnet, Weatherspoon, \& Hoehn, 2008; Silveira et al., 2016). Both roasters, according to The World Coffee Research Sensory Lexicon (2017), gave sensory characteristic 
Main characteristics of the samples

\begin{tabular}{|l|l|l|l|l|}
\hline Sample name & \multicolumn{1}{|c|}{ Roastery } & \multicolumn{1}{c|}{ Origin } & \multicolumn{1}{c|}{ Roasting level } & \multicolumn{1}{c|}{ Sensory description from roasters } \\
\hline Honduras_1 & roastery 1 & Honduras & light-medium & dark plum, grapes, red pepper, toffee (caramel) \\
\hline Kenya & roastery 1 & Kenya & light-medium & blackberry, red pepper, roses, dark chocolate \\
\hline Columbia & roastery 1 & Columbia & light-medium & pineapple, dried apricot, elderflower \\
\hline Ethiopia & roastery 2 & Ethiopia & light-medium & lime, jasmine, chocolate cream, cherry brandy \\
\hline Honduras_2 & roastery 2 & Honduras & light-medium & dried fruits, passion fruit, melon, kombucha \\
\hline
\end{tabular}

descriptions and the descriptions are also stated on the specialty coffee label as sensory guidelines for consumers.

\section{Brewing method}

Coffee beans were immediately ground and prepared by French Press brewing technique $(150 \mathrm{~mL}$ of $93{ }^{\circ} \mathrm{C}$ water (Neptunas/Lithuania) was added to $16 \mathrm{~g}$ of coarse grind coffee, with extraction time: 4 minutes). Each sample after brewing was immediately analysed. All brewed coffee samples were prepared in duplicates.

Headspace Volatile Compound Extraction by SPME

Aroma compounds were extracted by solid phase microextraction (SPME). The coffee extract samples $(5 \mathrm{~mL})$ were transferred to $20 \mathrm{~mL}$ glass container. Extraction parameters: extraction temperature $+50 \pm 1{ }^{\circ} \mathrm{C}$; incubation time: 4 minutes; extraction time: 7 minutes. In the research four different fibres were used: divinylbenzene/ carboxen/ polydimethylsiloxane(DVB/CAR/PDMS), carboxen/ polydimethylsiloxane (CAR/PDMS), polydimethylsiloxane/ divinylbenzene (PDMS/DVB) and polyacrylate (PA). All fibres were purchased from Supelco (Bellefonte, PA, USA).

HS-SPME-GC-MS chromatographic parameters

'Perkin Elmer Clarus 500' chromatograph with mass spectrometer and 'Elite-Waw ETR' (60 mx $0,25 \mathrm{~mm}$ internal diameter; DF 0.25 column) were used for gas chromatography method. Injection parameters: desorption time -15 minutes; temperature $+250^{\circ} \mathrm{C}$. Working conditions were as follows: injector $250{ }^{\circ} \mathrm{C}$; transfer line to MSD $260{ }^{\circ} \mathrm{C}$; oven temperature start $40{ }^{\circ} \mathrm{C}$, hold $7 \mathrm{~min}$, programmed from $40-160{ }^{\circ} \mathrm{C}$ at $6{ }^{\circ} \mathrm{C} \mathrm{min}-1$ hold $10 \mathrm{~min}$, and from $160-210{ }^{\circ} \mathrm{C}$ at $10^{\circ} \mathrm{C} \mathrm{m^{-1 }}$, hold $15 \mathrm{~min}$. The column initial flow rate of $1 \mathrm{~mL} \mathrm{~min}^{-1}$ was held using helium as carrier gas. The outlet split 1:2 and between 40 and 300 mass-tocharge ratios were scanned. As a quantitative measure, the share in the total GC peak area for each compound is given. The compounds were identified using mass spectral database 'Nist98'(Gloess et al., 2013; Steen et al., 2017).

Statistical analysis

The data were express as mean. Analysis of variance was performed, and the significant differences were stated if $p<0.05$. The data were analysed with Microsoft Office Excel 2013.Principal component analysis (PCA) was used for reduction of variables to a smaller set called components. The first principal component demonstrates the highest variability, followed by second component with less variability. PCA was performed including volatile profile of all tested coffees. Data were treated using SPSS 23 software.

\section{Results and Discussion}

Evaluation of coffee volatile compound chemical groups, detected with different SPME fibres

From 16 chemical compound groups, 76 compounds were detected (compounds are summarized by their chemical groups in Table 2). CAR/PDMS fibre extracted significantly more volatile compounds compared to other fibres and showed the highest peak areas in all chemical compound groups, except for phenols. CAR/PDMS has shown lack of extracting phenol compounds from ground coffee also in Akiyama et al. (2007) study. The phenol compounds as 4-vinylguaiacol or 4-ethyl-2-methoxyphenol are not common compounds in specialty coffee aroma profile because of the smoky, spicy unpleasant aroma notes and increasing concentration is only detected in medium-dark roasted coffees (Dorfner et al., 2003; Yang et al., 2016). Roberts, Pollien, \& Milo, (2000) research also reports CAR/PDMS fibre as the most suitable for small molecule extraction as 2-methylpropanal, acetaldehyde and organic acid as acetic acid and propionic acid extraction. The CAR has a large surface area with different range pores (micro- and macro-pores), which allows extracting also trace level volatile compound, compared to DVB with limited micropores (Chen, Chiang, \& Chung, 2019; Salum et al., 2017). The results also confirm this statement because using DVB/CAR/PDMS and CAR/PDMS fibre volatile compounds showed a higher peak area range compared to PDMS/DVB. $\mathrm{DVB} / \mathrm{CAR} / \mathrm{PDMS}$ fibre is the most commonly chosen fibre for aroma analysis of coffee brews. However, these studies used distilled water for coffee extraction (Caporaso et al., 2018; Mestdagh et al., 2014). It is known that distilled water cannot extract beneficial 
Chemical compound groups extracted from coffee brews using four different SPME fibres

\begin{tabular}{|c|c|c|c|c|c|}
\hline \multirow[b]{2}{*}{ No. } & \multirow{2}{*}{$\begin{array}{l}\text { Chemical compound } \\
\text { groups (number of } \\
\text { compounds) }\end{array}$} & \multicolumn{4}{|c|}{ GC-MS peak area (number of compounds) } \\
\hline & & $\begin{array}{l}\text { DVB/CAR/PDMS } \\
\text { fibre }\end{array}$ & CAR/PDMS fibre & PDMS/DVB fibre & PA fibre \\
\hline 1. & Alcohols (7) & & $5.68 \mathrm{E}+07^{\mathrm{a}}(3)$ & $2.89 \mathrm{E}+07^{\mathrm{c}}(3)$ & $3.01 \mathrm{E}+07^{\mathrm{b}}(3)$ \\
\hline 2. & Aldehydes(11) & $3.18 \mathrm{E}+08^{b}(3)$ & $9.61 \mathrm{E}+08^{\mathrm{a}}(8)$ & $2.39 \mathrm{E}+08^{\mathrm{c}}(6)$ & $3.07 \mathrm{E}+07(5)$ \\
\hline 3. & Alkanes (2) & $6.37 \mathrm{E}+06^{\mathrm{b}}(1)$ & $2.89 \mathrm{E}+07^{\mathrm{a}}(1)$ & & \\
\hline 4. & Ketones (9) & $6.71 \mathrm{E}+07^{\mathrm{b}}(4)$ & $2.95 \mathrm{E}+08^{\mathrm{a}}(7)$ & $4.17 \mathrm{E}+07(2)$ & $4.32 \mathrm{E}+05^{\mathrm{c}}(1)$ \\
\hline 5. & Amides (1) & & $9.61 \mathrm{E}+07(1)$ & & \\
\hline 6. & Esters $(8)$ & $7.09 \mathrm{E}+07^{\mathrm{b}}(5)$ & $3.19 \mathrm{E}+08^{\mathrm{a}}(6)$ & $1.53 \mathrm{E}+07^{c}(1)$ & \\
\hline 7. & Furans (6) & $4.09 \mathrm{E}+08^{\mathrm{b}}(5)$ & $1.43 \mathrm{E}+09^{\mathrm{a}}(6)$ & $3.97 \mathrm{E}+08^{\mathrm{c}}(4)$ & $8.46 \mathrm{E}+07(3)$ \\
\hline 8. & Organic acids (10) & $1.90 \mathrm{E}+07^{\mathrm{c}}(4)$ & $3.07 \mathrm{E}+08^{\mathrm{a}}(3)$ & $1.02 \mathrm{E}+07(5)$ & $3.02 \mathrm{E}+07^{\mathrm{b}}(2)$ \\
\hline 9. & Oxides (1) & $1.12 \mathrm{E}+07(1)$ & $1.02 \mathrm{E}+08^{\mathrm{a}}(1)$ & $1.69 \mathrm{E}+07^{\mathrm{c}}(1)$ & $1.01 \mathrm{E}+08^{\mathrm{b}}(1)$ \\
\hline 10. & Phenols (3) & & $4.77 \mathrm{E}+05^{\mathrm{c}}(1)$ & $3.50 \mathrm{E}+06^{\mathrm{b}}(1)$ & $3.72 \mathrm{E}+06^{\mathrm{a}}(1)$ \\
\hline 11. & Pyrazines (9) & $7.96 \mathrm{E}+06(1)$ & $1.13 \mathrm{E}+08^{\mathrm{a}}(7)$ & $4.10 \mathrm{E}+07^{\mathrm{b}}(6)$ & \\
\hline 12. & Pyridine (1) & $2.37 \mathrm{E}+07^{\mathrm{b}}(1)$ & $7.03 \mathrm{E}+07^{\mathrm{a}}(1)$ & $2.47 \mathrm{E}+06(1)$ & \\
\hline 13. & Pyrimidine (2) & $3.33 \mathrm{E}+07^{\mathrm{b}}(1)$ & $8.07 \mathrm{E}+07^{\mathrm{a}}(1)$ & $4.67 \mathrm{E}+06^{\mathrm{c}}(1)$ & $5.10 \mathrm{E}+05(1)$ \\
\hline 14. & Pyrroles (2) & $1.20 \mathrm{E}+07^{\mathrm{c}}(2)$ & $5.21 \mathrm{E}+07^{\mathrm{a}}(2)$ & $2.87 \mathrm{E}+07^{\mathrm{b}}(1)$ & \\
\hline 15. & $\begin{array}{l}\text { Sulfur containing } \\
\text { compounds (2) }\end{array}$ & & $1.77 \mathrm{E}+07^{\mathrm{a}}(1)$ & $2.59 \mathrm{E}+06^{\mathrm{b}}(1)$ & \\
\hline 16. & Terpenes (2) & & $1.56 \mathrm{E}+07^{\mathrm{a}}(1)$ & $3.41 \mathrm{E}+06^{\mathrm{b}}(2)$ & \\
\hline \multicolumn{2}{|c|}{ Total compounds (76) } & $9.79 \mathrm{E}+08^{\mathrm{b}}(28)$ & $3.94 \mathrm{E}+09^{\mathrm{a}}(50)$ & $8.33 \mathrm{E}+08^{\mathrm{c}}(35)$ & $2.81 \mathrm{E}+08(17)$ \\
\hline
\end{tabular}

Different letters $(\mathrm{a}-\mathrm{c})$ in the same row indicate significant differences $(\mathrm{p}<0.05)$ in $\mathrm{GC}-\mathrm{MS}$ peak area.

aroma and flavour compounds because of the lack of minerals like calcium or magnesium (Hendon, Colonna-Dashwood, \& Colonna-Dashwood, 2014). From this point of view, DVB/CAR/PDMS fibre suitability for coffee brew aroma extractions was not proven.

The PA fibre was able to extract only 17 volatile compounds from 8 chemical compound groups, showing the lowest total compound peak area, but the advantage of current fibre is the extraction of phenols with a higher peak area. It has been reported that fibre coatings with polar and non-polar materials are more efficient for polar compound extraction from samples with polar matrix composition. It could be one of the reasons why using PA fibre alkanes, amides, esters, pyrazines, pyridines, pyrroles, sulphur containing compounds and terpenes were not detected. Chen, Chiang, \& Chung, (2019) study also confirmed that using PA fibre compounds as ketones, pyrazines, sulphur containing compounds were not detected. The DVB/CAR/PDMS, CAR/PDMS and PDMS/DVB fibres are bipolar and the results confirm their ability to extract wider composition of volatile compounds (Mestdagh et al., 2014; Spietelun et al., 2010).

Important volatile compounds in specialty coffee brew

It is noted that from approximately 950 coffee volatile compounds only 20 can significantly influence the aroma and flavour profile. These 20 compounds are mostly detected in specialty coffees (Kim et al., 2018; Laukaleja \& Kruma, 2018). In this study, using CAR/PDMS fibre 17 important specialty coffee volatile compounds were detected: DVB/CAR/ PDMS detected 15, PDMS/DVB detected 13 and PA fibre only detected 6 compounds. A higher peak area of volatile compounds was detected using CAR/ PDMS and DVB/CAR/ PDMS fibres (the results are illustrated in Table 3). Both fibres detected a significantly higher peak area for 2,6-dimethyl-4thiopyrone (caramel aroma notes), 2-methylfuran (roasted coffee aroma notes). The CAR/PDMS fibre is suggested for detection of compounds with low boiling point, especially aldehydes (Salum et al., 2017). In this research, the CAR/PDMS was the only fibre which could extract acetaldehyde (fruity aroma notes) and had a higher peak area for 3methylbutanal (fruity), 2-methylpropanal (roasted, sweet almond aroma notes). The overall volatile compound profile in this research corroborates with Wei et al. (2017) aroma analysis using CAR/PDMS fibre; the furan and aldehyde compound peak areas were similar and proportional. Petisca et al. (2012) study reports supporting evidence that CAR/PDMS is the most efficient for furan compound extraction from coffee. The DVB/CAR/PDMS was the only 
Table 3

Identified important volatile compounds in specialty coffee brews using four different SPME fibres

\begin{tabular}{|c|c|c|c|c|c|c|}
\hline \multirow{2}{*}{ No. } & \multirow{2}{*}{ Compounds } & \multicolumn{4}{|c|}{ GC-MS peak area $\left(\times 10^{8}\right)$} & \multirow[t]{2}{*}{$\begin{array}{c}\text { Compound sensory } \\
\text { description }\end{array}$} \\
\hline & & $\begin{array}{l}\text { DVB/CAR/ } \\
\text { PDMS }\end{array}$ & $\begin{array}{l}\text { CAR/ } \\
\text { PDMS }\end{array}$ & $\begin{array}{l}\text { PDMS/ } \\
\text { DVB }\end{array}$ & PA & \\
\hline 1. & $\begin{array}{l}\text { 1-(2-Furanylmethyl })-1 \mathrm{H}- \\
\text { pyrrole }\end{array}$ & $19.64^{\mathrm{b}}$ & $48.81^{\mathrm{a}}$ & $7.08^{\mathrm{c}}$ & & savoury, vegetables \\
\hline 2. & 2,3-Pentanedione & 5.01 & & & & buttery \\
\hline 3. & 2,6-Dimethyl-4-thiopyrone & $56.01^{\mathrm{b}}$ & $127.43^{\mathrm{a}}$ & $33.73^{\mathrm{c}}$ & 5.01 & caramel \\
\hline 4. & $\begin{array}{l}\text { 5-Methyl-2- } \\
\text { furancarboxaldehyde }\end{array}$ & $35.85^{\mathrm{b}}$ & $73.31^{\mathrm{a}}$ & $20.29^{c}$ & 10.35 & caramel \\
\hline 5. & 2-Furanmethanol & $60.46^{\mathrm{a}}$ & & $42.15^{b}$ & $3.66^{\mathrm{c}}$ & floral \\
\hline 6. & Furfuryl acetate & $27.37^{\mathrm{b}}$ & $34.29^{\mathrm{a}}$ & $11.89^{\mathrm{c}}$ & 3.06 & floral, fruity \\
\hline 7. & 3-Methyl-butanal & $7.14^{\mathrm{b}}$ & $12.39^{\mathrm{a}}$ & $6.57^{\mathrm{c}}$ & 0.86 & fruity \\
\hline 8. & 1-(2-Furanyl), ethanone & $7.85^{\mathrm{a}}$ & $3.29^{\mathrm{b}}$ & & & nutty \\
\hline 9. & 2-(Methoxymethyl)furan & $95.72^{\mathrm{a}}$ & $34.00^{\mathrm{b}}$ & 2.93 & & nutty, ground coffee-like \\
\hline 10. & 2-Methylfuran & $73.77^{\mathrm{b}}$ & $164.31^{\mathrm{a}}$ & $27.06^{\mathrm{c}}$ & 6.86 & roasted almond, coffee \\
\hline 11. & Furfural & $14.57^{\mathrm{b}}$ & $16.84^{\mathrm{a}}$ & $5.16^{\mathrm{c}}$ & & caramel \\
\hline 12. & 2-Methylpropanal & & $3.98^{\mathrm{a}}$ & $3.28^{\mathrm{b}}$ & & roasted sweet almond \\
\hline 13. & 2.5-Dimethylpyrazine & $7.96^{\mathrm{a}}$ & $5.48^{\mathrm{b}}$ & $3.56^{\mathrm{c}}$ & & roasted \\
\hline 14. & Ethyl-pyrazine & $16.63^{\mathrm{b}}$ & $32.29^{\mathrm{a}}$ & $9.35^{\mathrm{c}}$ & & chocolate-peanut, nutty \\
\hline 15. & 4-Methyl-pyrimidine & & 18.20 & & & popcorn \\
\hline 16. & Acetaldehyde & & 6.15 & & & fruity \\
\hline 17. & Furfurylformate & $19.64^{\mathrm{b}}$ & $48.81^{\mathrm{a}}$ & $7.08^{\mathrm{c}}$ & & malt, fruity \\
\hline
\end{tabular}

Different letters $(a-c)$ in the same row indicate significant differences $(\mathrm{p}<0.05)$ in GC-MS peak area.

fibre which could detect 2,3-pentanedione (buttery aroma notes) in coffee brews and showed the highest peak area for 2-furanmethanol (floral aroma notes), 2-(methoxymethyl) furan (nutty, ground coffee like aroma notes), 2,5-dimethylpyrazine (roasted aroma notes) compounds.

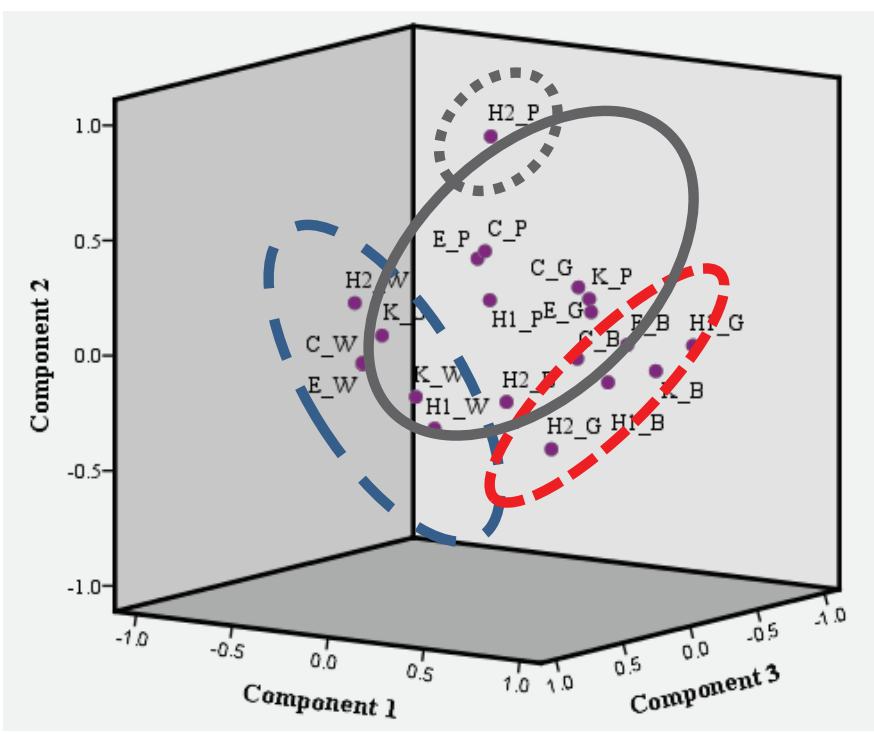

Figure 1. Component Plot in Rotated Space by principal component analysis (PCA). Code names: E_W - Ethiopia PA fibre; C_W - Columbia PA fibre; H1_W - Honduras_1 PA fibre; H2_W - Honduras_2 PA fibre; K_W - Kenya PA fibre; E_P - Ethiopia PDMS/DVB fibre; C_P - Columbia PDMS/DVB fibre; H1_P - Honduras_1 PDMS/DVB fibre; H2_P Honduras_2 PDMS/DVB fibre; K_P - Kenya PDMS/DVB fibre; E_B - Ethiopia CAR/PDMS fibre; C_B - Columbia CAR/ PDMS fibre; H1_B - Honduras_1 CAR/PDMS fibre; H2_B - Honduras_2 CAR/PDMS fibre; K_B - Kenya CAR/PDMS fibre; E_G - Ethiopia DVB/CAR/PDMS fibre; C_G - Columbia DVB/CAR/PDMS fibre; H1_G - Honduras_1 DVB/CAR/ PDMS fibre; H2_G - Honduras_2 DVB/CAR/PDMS fibre; K_G - Kenya DVB/CAR/PDMS fibre. 
This study result disproves Bressanello et al. (2017) and Gloess et al. (2013) researches; they reported a positive correlation between sensory (cupping) results and important coffee volatile compounds determined by HS-SPME using PDMS/DVB. Ribeiro et al. (2009) using PDMS/DVB fibre for prediction of sensory properties of Brazilian Coffea arabica $L$. roasted coffees concluded the importance of the method for future alternative coffee beverage quality monitoring. PCA of the specialty coffee brews with different fibres

The Principal component analysis reduced variables in components and the first two components represented $83.84 \%$ of total variance. In the score plot, coffee samples extracted with Carboxen/PDMS (CAR/PDMS) and Divinylbenzene/Carboxen/ PDMS (DVB/CAR/PDMS) were classified in one principal component showing the highest content of the most important coffee volatiles (Figure 1). In separate principal components, samples extracted with polyacrylate (PA) and Polydimethylsiloxane/ Divinylbenzene (PDMS/DVB) fibre were classified. Due to the specific volatile profile, Honduras_2 samples were classified differently - the sample extracted with Carboxen/PDMS was classified together with other coffee samples extracted with Polydimethylsiloxane/Divinylbenzene (PDMS/ DVB), and the Honduras_2 sample, extracted with Polydimethylsiloxane/Divinylbenzene (PDMS/ DVB), was classified separately from all tested samples. The results can be explained by the applied processing method. Honduras_2 was the only coffee sample with different processing method (anaerobic), while for the other four coffees natural processing method was applied. Natural process method allows the coffee cherries to dry in the sun, while in anaerobic process coffee cherries are washed and coffee beans are afterwards sealed in fermentation tanks. During the anaerobic process coffee develops the fermented aroma and flavour notes (Geromel et al., 2008; Taveira et al., 2015).

The Principal component analysis allowed to reduce data of coffee volatiles in principal components, and results confirmed the importance of SPME fibre coating selection for analysis of coffee volatile profile, because samples were classified based on fibre coating, not the origin of coffees.

\section{Conclusions}

The results demonstrated that CAR/PDMS, compared to other fibres, was able to extract significantly more volatile compounds with higher peak areas in all chemical compound groups, except phenols. The CAR/PDMS was the only fibre which could detect all 17 important volatile compounds for specialty coffee. The PA fibre showed the largest phenol peak area but could only detect 6 from 17 important volatile compounds. This research disproves previous reports, showing that PDMS/DVB and DVB/CAR/PDMS fibres detect a wider and higher concentration of coffee volatile compounds compared to CAR/PDMS fibre. The result provides evidence for specialty coffee aroma profile analysis by SPME.

\section{Acknowledgements}

Present research has been supported by the program 'Strengthening Research Capacity in the Latvia University of Life Sciences and Technologies' project 'The changes of biologically active compounds of Specialty coffee under the influence of technological processes' (Z22).

\section{References}

1. Akiyama, M., Murakami, K., Ikeda, M., Iwatsuki, K., Wada, A., Tokuno, K., ... Iwabuchi, H. (2007). Analysis of the Headspace Volatiles of Freshly Brewed Arabica Coffee Using Solid-Phase Microextraction. Food Chemistry and Toxicology. 72(7), 388-396. DOI: 10.1111/j.1750-3841.2007.00447.x.

2. Bressanello, D., Liberto, E., Cordero, C., Rubiolo, P., Pellegrino, G., Ruosi, M.R., \& Bicchi, C. (2017). Coffee aroma: Chemometric comparison of the chemical information provided by three different samplings combined with GC-MS to describe the sensory properties in cup. Food Chemistry. 214(Supplement C), 218-226. DOI: 10.1016/j.foodchem.2016.07.088.

3. Caporaso, N., Whitworth, M.B., Cui, C., \& Fisk, I.D. (2018). Variability of single bean coffee volatile compounds of Arabica and robusta roasted coffees analysed by SPME-GC-MS. Food Research International. 108(April), 628-640. DOI: 10.1016/j.foodres.2018.03.077.

4. Chen, Y.P., Chiang, T.K., \& Chung, H.Y. (2019). Optimization of a headspace solid-phase microextraction method to quantify volatile compounds in plain sufu, and application of the method in sample discrimination. Food Chemistry. 275 (April 2018), 32-40. DOI: 10.1016/j.foodchem.2018.09.018.

5. Donnet, M.L., Weatherspoon, D.D., \& Hoehn, J.P. (2008). Price determinants in top-quality e-auctioned specialty coffees. Agricultural Economics. 38(3), 267-276. DOI: 10.1111/j.1574-0862.2008.00298.x.

6. Dorfner, R., Ferge, T., Kettrup, A., Zimmermann, R., \& Yeretzian, C. (2003). Real-Time Monitoring of 4-Vinylguaiacol, Guaiacol, and Phenol during Coffee Roasting by Resonant Laser Ionization Time-ofFlight Mass Spectrometry. Journal of Agricultural and Food Chemistry. 51, 5768-5773. 
7. Fassio, L.O., Malta, M.R., Liska, G.R., Alvarenga, S.T., Sousa, M.M.M., Farias, T.R.T., \& Pereira, R.G.F.A. (2017). Sensory Profile and Chemical Composition of Specialty Coffees from Matas de Minas Gerais, Brazil. Journal of Agricultural Science. 9(9), 78. DOI: 10.5539/jas.v9n9p78.

8. Geromel, C., Ferreira, L.P., Davrieux, F., Guyot, B., Ribeyre, F., Brígida dos Santos Scholz, M., ... Marraccini, P. (2008). Effects of shade on the development and sugar metabolism of coffee (Coffea arabica L.) fruits. Plant Physiology and Biochemistry. 46(5-6), 569-579. DOI: 10.1016/j.plaphy.2008.02.006.

9. Gloess, A.N., Schönbächler, B., Klopprogge, B., D’Ambrosio, L., Chatelain, K., Bongartz, A., ... Yeretzian, C. (2013). Comparison of nine common coffee extraction methods: Instrumental and sensory analysis. European Food Research and Technology. 236(4), 607-627. DOI: 10.1007/s00217-013-1917-X.

10. Hendon, C.H., Colonna-Dashwood, L., \& Colonna-Dashwood, M. (2014). The Role of Dissolved Cations in Co ffee Extraction. Journal of Agricultural and Food Chemistry. 62, 4947-4950.

11. Kim, S., Ko, J., Kang, B., \& Park, H. (2018). Prediction of key aroma development in coffees roasted to diff erent degrees by colorimetric sensor array, 240(July 2017), 808-816. DOI: 10.1016/j. foodchem.2017.07.139.

12. Laukaleja, I., \& Kruma, Z. (2018). Quality of Specialty Coffee: Balance between aroma, flavour and biologically active compound composition: Review. In Research for Rural Development Vol. 1, DOI: 10.22616/rrd.24.2018.038.

13. Lee, K.H., Bonn, M.A., \& Cho, M. (2013). Consumer motives for purchasing organic coffee. The moderating effects of ethical concern and price sensitivity. International Journal of Contemporary Hospitality Management. 27(6), 1157-1180. DOI: 10.1108/MRR-09-2015-0216.

14. Lee, S.J., Kim, M.K., \& Lee, K.G. (2017). Effect of reversed coffee grinding and roasting process on physicochemical properties including volatile compound profiles. Innovative Food Science and Emerging Technologies. 44(October 2016), 97-102. DOI: 10.1016/j.ifset.2017.07.009.

15. Mestdagh, F., Davidek, T., Chaumonteuil, M., Folmer, B., \& Blank, I. (2014). The kinetics of coffee aroma extraction. Food Research International. 63, 271-274. DOI: 10.1016/j.foodres.2014.03.011.

16. Petisca, C., Pérez-Palacios, T., Farah, A., Pinho, O., \& Ferreira, I.M.P.L.V.O. (2012). Furans and other volatile compounds in ground roasted and espresso coffee using headspace solid-phase microextraction : Effect of roasting speed. Food and Bioproducts Processing. (July), 1-9. DOI: 10.1016/j.fbp.2012.10.003.

17. Piccino, S., Boulanger, R., Descroix, F., \& Sing, A.S.C. (2014). Aromatic composition and potent odorants of the "specialty coffee" brew "Bourbon Pointu" correlated to its three trade classifications. Food Research International. 61, 264-271. DOI: 10.1016/j.foodres.2013.07.034.

18. Poltronieri, P., \& Rossi, F. (2016). Challenges in Specialty Coffee Processing and Quality Assurance. Challenges. 7(19), 1-22. DOI: 10.3390/challe7020019.

19. Ribeiro, J.S., Augusto, F., Salva, T.J.G., Thomaziello, R.A., \& Ferreira, M.M.C. (2009). Prediction of sensory properties of Brazilian Arabica roasted coffees by headspace solid phase microextractiongas chromatography and partial least squares. Analytica Chimica Acta. 634, 172-179. DOI: 10.1016/j. aca.2008.12.028.

20. Roberts, D.D., Pollien, P., \& Milo, C. (2000). Solid-Phase Microextraction Method Development for Headspace Analysis of Volatile Flavor Compounds. Journal of Agricultural and Food Chemistry. 48, 2430-2437.

21. Salum, P., Erbay, Z., Kelebek, H., \& Selli, S. (2017). Optimization ofHeadspace Solid-Phase Microextraction with Different Fibers for the Analysis of Volatile Compounds of White-Brined Cheese by Using Response Surface Methodology. Food Analytical Methods. 10(6), 1956-1964. DOI: 10.1007/s12161-016-0774-1.

22. Silveira, A. de S., Pinheiro, A.C.T., Ferreira, W.P.M., Silva, L.J. da, Rufino, J.L. dos S., \& Sakiyama, N.S. (2016). Sensory analysis of specialty coffee from different environmental conditions in the region of Matas de Minas, Minas Gerais, Brazil. Revista Ceres. 63(4), 436-443. DOI: 10.1590/0034-737X201663040002.

23. Spietelun, A., Pilarczyk, M., Kloskowski, A., \& Namieśnik, J. (2010). Current trends in solid-phase microextraction (SPME) fibre coatings. Chemical Society Reviews. 39(11), 4524-4537. DOI: 10.1039/ c003335a.

24. Steen, I., Waehrens, S.S., Petersen, M.A., Münchow, M., \& Bredie, W.L.P. (2017). Influence of serving temperature on flavour perception and release of Bourbon Caturra coffee. Food Chemistry. 219, 61-68. DOI: 10.1016/j.foodchem.2016.09.113.

25. Taveira, J.H.D.S., Sttela, D.V.F.D.R., Eder, P.I., Pedro, D.O., \& Gerson, S.G. (2015). Post-harvest effects on beverage quality and physiological performance of coffee beans. African Journal of Agricultural Research. 10(12), 1457-1466. DOI: 10.5897/ajar2014.9263. 
26. The World Coffee (2017). The World Coffee Research Sensory Lexicon. Retrieved March 10, 2019, from https://worldcoffeeresearch.org/media/documents/20170622_WCR_Sensory_Lexicon_2-0.pdf.

27. Wei, L., Liu, X., San, W., Wong, E., \& Quan, S. (2017). Food Hydrocolloids. Effects of sucrose monopalmitate (P90), Tween 80 and modi fi ed starch on coffee aroma retention and release in coffee oilbased emulsions. Food Hydrocolloids. 66, 128-135. DOI: 10.1016/j.foodhyd.2016.12.021.

28. Yang, N., Liu, C., Liu, X., Degn, T.K., Munchow, M., \& Fisk, I. (2016). Determination of volatile marker compounds of common coffee roast defects. Food Chemistry. 211, 206-214. DOI: 10.1016/j. foodchem.2016.04.124. 